\title{
Analysis of chromatin pattern in blood lymphocytes of healthy donors and in lymphoid cells of patients with chronic lymphocytic leukaemia
}

\author{
M RÓZYCKA, W SAWICKI, Z TRACZYK, * W BEM, P STROJNY \\ From the Department of Histology and Embryology, Institute of Biostructure, Medical School, Warsaw, and the \\ *Fourth Division of Internal Medicine, Central Clinical Hospital, Warsaw, Poland
}

SUMMARY The optical Fourier transformation was used to analyse the chromatin/interchromatin pattern of lymphocytes of healthy subjects and lymphoid cells of patients with chronic lymphocytic leukaemia (CLL, type B, stage O). Peripheral blood smears were prepared routinely, fixed, and stained by the Feulgen method, and the photographic images of the nuclei were quantitatively analysed. From the radial distribution of light intensity of diffractograms, several Feulgen chromatin (F-chromatin/interchromatin) descriptors were evaluated. Four showed the strongest discriminant power and these descriptors discriminated well between lymphocytes of healthy donors and lymphoid cells of CLL patients, although F-chromatin/interchromatin components of the same sizes were found in lymphocytes and lymphoid cells.

As the chromatin pattern in nuclei is related to cell function, ${ }^{12}$ this feature of fixed and stained cells can be successfully used for discrimination between cells. Various chromatin pattern descriptors have been used, although the optical density of stained nuclei is most often analysed..$^{3-5}$ The modification of chromatin pattern during the cell cycle and terminal differentiation $^{6-8}$ have been reported. Recently, the chromatin pattern was investigated in a number of extensive studies of sectioned lymphoid cells from lymph nodes of non-Hodgkin's lymphomas to determine the best discriminant. ${ }^{39-13}$ Morphometric methods were applied, which included the following variables: form factor, surface area of nucleus and cell, and nucleolar contour index, as well as texture analysis with estimation of nuclear elongation, range of widths of blocks of chromatin and their distribution along the nuclear contour. The results of these studies showed the usefulness of such an analysis for differentiating the various categories of non-Hodgkins's lymphoma.

Optical Fourier analysis of fixed and stained nuclei offers a quantitative assessment of their chromatin/ interchromatin pattern. Though not densitometric, optical Fourier analysis may indicate the extent of variously sized components of F-chromatin/interchromatin of normal lymphocytes and lymphoid cells in chronic lymphocytic leukaemia (CLL).

This study aimed to apply optical Fourier analysis

Accepted for publication 30 September 1987 of Feulgen-stained nuclei of lymphocytes or lymphoid cells of the peripheral blood smears obtained from healthy adult subjects and patients with CLL type B. stage $\mathbf{O}$.

\section{Material and methods}

Peripheral blood smears from five healthy subjects aged between 20 and 30 years and four patients with confirmed chronic lymphocytic leukaemia (CLL. type $\mathrm{B}$, stage $\mathrm{O}^{14}$ ) were examined. Diagnosis of CLL was based on cytological, immunological, and clinical criteria. ${ }^{15}$ The blood smears were prepared as gently as possible to avoid damaging the lymphoid cells. ${ }^{16}$ Smears were fixed in three parts $96 \% \mathrm{v} / \mathrm{v}$ ethanol and one part acetone for 10 minutes, rinsed with ethanol for five minutes, and dried in air. The fixed smears were Feulgen stained by hydrolysis at $19^{\circ} \mathrm{C}$ for 1.5 hours in $5 \mathrm{~N}$ hydrochloric acid and treatment in Schiff's reagent (basic fuchsin, BDH Chemical Limited, Poole, England) for 1.5 hours at $19^{\circ} \mathrm{C} . .^{17}$

OPTICAL DIFFRACTOMETRY

Between 11 and 25 randomly chosen nuclei from each donor were photographed (objective $\times 100$, eye piece $\times 10$, Foton Super-Orto negative film, 12 DIN, gamma coefficient $=1 \cdot 4$ ), yielding 87 images of nuclei from healthy subjects and 90 of patients with CLL. To prevent modification of film contrast due to the 
photographic technique, an identical procedure was followed in each case.

Diffraction patterns from the microphotographs of nuclei were obtained by means of a diffractometer equipped with a helium-neon laser operating at a wave length of $633 \mathrm{~nm}$ (Centralne Laboratorium Optyki, Warsaw, Poland). ${ }^{18}$ The light intensity of the diffractographic pattern was recorded by an electronic detector type DPSU-2 (Recognition System Inc., Van Nuys, California, USA) with a detector plate (WRD $6400 \mathrm{~A})$ consisting of 32 ring- and 32 wedge-shaped elements. In each ring the light intensity was integrated in specified ranges of spatial frequency (radial light intensity distribution). The outputs of all photodiodes were converted from analogue to digital form and processed by minicomputer.

\section{MATHEMATICAL EVALUATION}

To minimise errors of diffractographic measurements, the radial light intensity distribution of the diffraction pattern of each analysed nucleus was modified according to the following formula':

$$
\mathrm{X}_{\mathrm{i}}=\frac{\mathrm{K}_{\mathrm{i}}}{\sum_{\mathrm{i}=1}^{32} \mathrm{~K}_{\mathrm{i}}}
$$

where: $\quad \mathrm{K}_{\mathrm{i}}=\left(\mathrm{x}_{\mathrm{i}}-\mathrm{Y}\right) / \mathrm{C}_{\mathrm{i}} ; \quad \mathrm{x}_{\mathrm{i}}=$ light intensity measured in the "i" ring of the detector; $Y=$ electronic "noise" in the detecting system; $\mathrm{C}_{\mathrm{i}}=$ correction coefficient given by the manufacturer for the " $\mathrm{i}$ " ring of the detector.

The division of $\mathrm{K}_{\mathrm{i}}$ by $\sum_{\mathrm{i}}^{32} \mathrm{~K}_{\mathrm{i}}$-that is, normalisation of values of light intensity measured in a given ring in relation to total light intensity measured in the diffractograms-eliminates errors of instability of laser light and of variability in the contrast of various photomicrographs. This normalisation provides the information on the light intensity for each unit surface area of nucleus and the light intensity therefore becomes independent of the nucleus size.

Normalised radial light intensity distribution of each nucleus was quantitatively characterised by four descriptors: (i) the sum of light intensity measured in three rings closest to the detector center (SUM descriptor); (ii) the value of the tangent describing the slope of the distribution (TGA descriptor); (iii) the maximal value of spatial frequency at which the peaks of light intensity distribution ceased (B descriptor) and; (iv) the value of light intensity at the level at which the peaks of light intensity distribution ceased (the background level, XLEV descriptor).

For each photomicrograph of each nucleus two measurements of radial light intensity distribution were carried out: one included and another one failed to include the nuclear image (and so acted as a blank). For each detector ring, the normalised value of light intensity which carried information of the nuclear image was divided by the respective value which failed to carry the information of the nuclear image. This procedure, explained in detail elsewhere ${ }^{20}$ intensified the signal:noise ratio.

In modified distribution the peaks of light intensity were identified and their number ( $\mathrm{N}$ descriptor), height (light intensity, $\mathrm{MAX}_{\mathrm{i}=1 . \mathrm{N}}$ descriptor), position (spatial frequency, IMAX $\mathrm{I}_{\mathrm{j}=1 \ldots \mathrm{N}}$ descriptor), and half-width (spatial frequency, WIDTH descriptor) were obtained. In addition, the minima of radial light intensity distribution were identified and their level of light intensity $\left(\mathrm{MIN}_{\mathrm{j}=1, \mathrm{~N}-\text {, }}\right.$ parameter) and position (spatial frequency, $I M I N_{j=1}, N_{-1}$ descriptor) were also obtained. Moreover, the mean value of radial light intensity was calculated (MEAN descriptor) according to the following formula as well as its standard deviation (SD variable).

$$
\operatorname{MEAN}=\frac{\sum_{\mathrm{i}=1}^{32}\left(\frac{\mathrm{X}_{\mathrm{i}}}{\mathrm{E}_{\mathrm{i}}}\right)}{32}
$$

where: $X_{i}=$ the value of normalised light intensity carrying the information of the nuclear image measured in the " $i$ " ring of detector; $E_{i}=$ the value of normalised light intensity which failed to carry information of the nuclear image.

Finally, the normalised radial light intensity distribution of each nucleus was characterised by several continuous values of light intensity (SUM, TGA, XLEV, MAX , MIN $_{\mathrm{j}}$, MEAN, SD) and several discrete values of spatial frequency IMAX $_{j}$, WIDTH, $\mathrm{IMIN}_{\mathrm{j}}$ ). All descriptors were regarded as the variables evaluated either by Mann-Whitney non-parametric test (descriptors of discrete values) or by variance analysis (descriptors of continuous values). By the step-wise method based on Wilks's criterion ${ }^{21}$ diffractographic descriptors with the greatest discriminant power were selected. The selected descriptors were: (i) the mean value of light intensity of the diffractographic pattern obtained according to the second formula (MEAN descriptor); (ii) standard deviation of the mean value of light intensity of the diffractographic pattern (SD descriptor); (iii) maximal value of the light intensity in the radial light intensity distribution (MAX descriptor); and (iv) the level of the background (XLEV descriptor).

The four selected diffractographic descriptors were linearly combined and formed a discriminant function..$^{22}$ Discriminant score was counted for each diffractographic pattern-that is, for each cell nucleus - and probability of classification of each particular nucleus was calculated as either that of a lymphocyte or of a lymphoid cell.

The mean positions of the peaks of the light intensity distribution (separately for nuclei of 
lymphocytes and lymphoid cells) were calculated as weighted means. The latter included the frequency of occurrence of a peak of light intensity at a given position of the distribution - that is, the number of distributions containing a peak for a given position at the abscissa-calculated separately for nuclei of lymphocytes and those of lymphoid cells. The size of chromatin/interchromatin components (d) was calculated from the mean radial position (spatial frequency) of the distribution peak according to the following formula:

$$
\mathrm{d}=\frac{1}{\mathrm{SF} \times \mathrm{Q}}
$$

where: $\mathrm{SF}=$ spatial frequency (lines $/ \mathrm{mm}$ ) for a given radial position: $Q=$ overall magnification of $a$ nucleus microphotograph.

The frequency of occurrence of F-chromatin/interchromatin components of a given size-that is, the number of nuclei in which components of $F$ chromatin/interchromatin of a given size were detected in relation to all analysed nuclei-in lymphocytes and lymphoid cells was compared by the $\chi^{2}$ test.

The normalised light intensity of diffractographic pattern depends chiefly on the optical density and the arrangement of image elements and on the level of contrast between variously sized structures. ${ }^{23}$ Because of the stoichiometry of the Feulgen reaction (used in the present study) this contrast can be regarded as constant provided that the same DNA packing ratio persists in the chromatin components of a given size in various nuclei. Though diffractometry is not a densitometric method, the light intensity of the diffractographic pattern representing F-chromatin/ interchromatin components of the same size can reflect their relative number. The variations of the background level of radial light intensity distribution therefore reflect the modifications of the density of F-chromatin/interchromatin components of the size below light microscopic resolution.

The values of light intensity measured in the same radial position of the diffractographic patterns - that is, representing the same size of F-chromatin/interchromatin elements-were verified by non-parametric Mann-Whitney U test.

To measure the surface area of nuclear projections, the nuclear contours were drawn by a camera lucida coupled with the light microscope, and coordinates of 20-30 outline points for each contour were introduced by a sonic pen into the memory of computer.

The surface area of projections of nuclei of lymphocytes and lymphoid cells were compared by Student's $t$-test.

\section{Results}

A diffractographic pattern was obtained for the F-

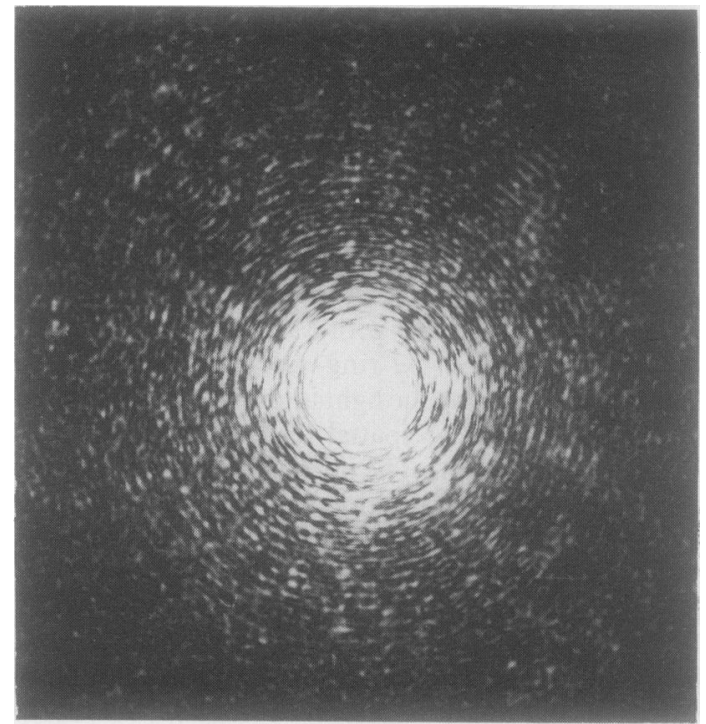

Fig 1 Typical diffractographic pattern of Feulgen-stained nucleus of lymphocyte of healthy subject.

chromatin/interchromatin image of each analysed nucleus of healthy subjects (lymphocyte nucleus) and CLL patients (lymphoid cell nucleus) (fig 1). Fig 2 shows the mean normalised radial light intensity distribution of lymphocyte and lymphoid cell nuclei $v$ F-chromatin/interchromatin component size calculated from spatial frequency.

To analyse the size of F-chromatin/interchromatin components which contribute to the modification of its pattern, the mean positions of the light intensity distribution peaks were calculated and converted to nanometers (third formula). The same ranges of component size were found for F-chromatin/interchromatin elements of lymphocytes and lymphoid cells: $360-406 \mathrm{~nm}, 845-960 \mathrm{~nm}, 2360-2650 \mathrm{~nm}, 3160$ $3600 \mathrm{~nm}$ and $4440-5290 \mathrm{~nm}$. The number of nuclei in

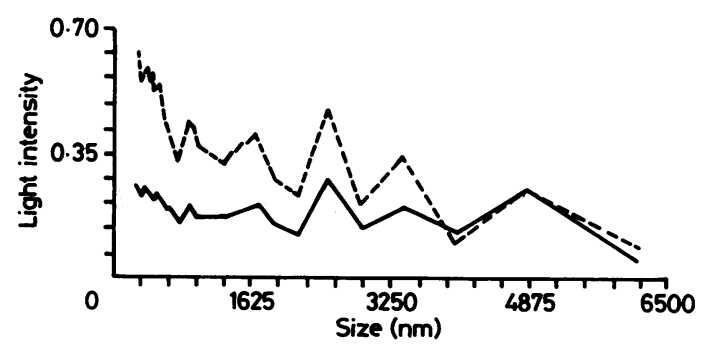

Fig 2 Normalised, mean radial light intensity distributions. Abscissa: F-chromatin/interchromatin component size calculated from spatial frequency. Solid line represents nuclei of lymphoid cells of CLL patients; dotted line represents nuclei of lymphocytes of healthy subjects. 
Table 1 Frequency* of occurrence of F-chromatin/ interchromatin components of various ranges of size calculated for nuclei of healthy subjects and CLL patients

\begin{tabular}{lll}
\hline \multirow{2}{*}{$\begin{array}{l}\text { Ranges of size } \\
(\mathrm{nm})\end{array}$} & \multicolumn{2}{l}{ Frequency of occurrence } \\
\cline { 2 - 3 } & Healthy subjects & CLL patients \\
\hline $360-406$ & $0.84 \dagger$ & 0.39 \\
$845-960$ & $0.86 \dagger$ & 0.60 \\
$2360-2650$ & 0.91 & 0.83 \\
$3160-3600$ & 0.58 & 0.48 \\
$4440-5190$ & $0.27 \dagger$ & 0.57 \\
\hline
\end{tabular}

*Frequency $=$ fraction of nuclei at which components of a given size appeared.

tsignificant difference $(x=0.05)$ between frequency values for healthy subjects and CLL patients.

which components of F-chromatin/interchromatin of a given size were detected in relation to all analysed nuclei and expressed as a fraction (referred to as frequency), separately for nuclei of lymphocytes and lymphoid cells, are presented in table 1 . The frequency of occurrence of F-chromatin/interchromatin components measuring $360-2650 \mathrm{~nm}$ held the level at $0 \cdot 8$ 0.9 for lymphocytes, while for lymphoid cells it was at the level of 0.4 (component size $360-406 \mathrm{~nm}$ ), rising $\left(\chi^{2}\right.$ test, $x=0.05)$ to 0.6 at component size $845-960 \mathrm{~nm}$ and to 0.8 at component size $2360-2650 \mathrm{~nm}$ - that is, reaching the level of those of lymphocytes). The frequency of occurrence of F-chromatin/interchromatin components measuring $3160-3600 \mathrm{~nm}$ and $4440-5290 \mathrm{~nm}$ was 0.6 for lymphocytes and 0.3 , while that for lymphoid cells was within the range $0.5-0.6-$ that is, at the level of the frequency of components measuring $845-960 \mathrm{~nm}\left(\chi^{2}\right.$ text, $\left.\alpha=0.05\right)$.

The distributions of the values of light intensity of diffraction patterns, representing the relative number of F-chromatin/interchromatin components, were non-Gaussian for lymphoid cells. The non parametric Mann-Whitney U test was therefore applied to verify data. The values of light intensity measured for the same range of F-chromatin/interchromatin component size were systematically higher $(\alpha=0.001)$ for lymphocytes than for lymphoid cells.

To analyse the F-chromatin/interchromatin pattern of lymphoid cell and lymphocyte nuclei, the radial

Table 2 Mean values of $M E A N, S D, M A X$, and $X L E V$ diffractographic descriptors for nuclei of healthy subjects and CLL patients

\begin{tabular}{lccccc}
\hline & \multicolumn{2}{c}{ Healthy subjects $(n=87)$} & & \multicolumn{2}{c}{ CLL patients $(n=90)$} \\
\cline { 2 - 3 } Descriptor & Mean & $S E M$ & & Mean & $S E M$ \\
\hline MEAN & 5.12 & 0.20 & & 2.70 & 0.12 \\
SD & 0.83 & 0.03 & & 0.47 & 0.03 \\
MAX & 27.60 & 2.90 & & 16.20 & 0.90 \\
XLEV & 0.0024 & 0.0002 & & 0.0016 & 0.0002 \\
\hline
\end{tabular}

Table 3 Discriminant analysis: percentage classification of lymphocyte nuclei of healthy subjects and those of $C L L$ patients as either "healthy" or CLL cells

\begin{tabular}{llll}
\hline & & \multicolumn{2}{l}{ Classified as: } \\
\cline { 3 - 4 } & No $(\%)$ & "Healthy"cells & CLL cells \\
\hline Healthy subjects & $87(100)$ & $80.5 \%$ & $19.5 \%$ \\
CLL patients & $90(100)$ & $12.3 \%$ & $87.7 \%$ \\
\hline
\end{tabular}

distributions of light intensity of diffractographic patterns were mathematically evaluated. The evaluation included calculation of a number of diffractographic descriptors of radial light intensity distribution and use of either variance analysis or Mann-Whitney U test. Finally, four descriptors of the highest discriminant power were selected as the best discriminators of the distributions of light intensity of diffractographic patterns of lymphocyte nuclei compared with those of lymphoid cell nuclei. The four descriptors (MEAN, SD, MAX, and XLEV) characterise in quantitative terms the compactness/ dispersion of nucleus content. Table 2 shows the mean values of MEAN, SD, MAX, and XLEV descriptors separately for F-chromatin/interchromatin of lymphocytes of healthy subjects and those of lymphoid cells of CLL patients. The mean values of all descriptors were significantly $(\alpha=0.001)$ lower for lymphoid cells than for lymphocytes.

The linear discriminant analysis applied to four selected descriptors showed the percentage of classification of a particular cell as a lymphocyte or lymphoid cell (table 3 ). About $20 \%$ of lymphocytes of healthy subjects had the F-chromatin/interchromatin features of lymphoid cells while about $11 \%$ of lymphoid cells of CLL patients had the features of lymphocytes of healthy subjects.

The mean value of the surface area of lymphocyte nuclear projection was found to be $\mathbf{4 0}$ (SEM $0 \cdot 5) \mu \mathrm{m}^{2}$ (the mean nuclear radius of $3570 \mathrm{~nm}$ ), while the mean surface area of the nuclear projection for lymphoid cells was $70(2) \mu \mathrm{m}^{2}$ (mean nuclear radius of $4720 \mathrm{~nm}$ ).

\section{Discussion}

The small number of blood donors (five healthy subjects and four CLL patients) investigated means that the results of this study require further evaluation but it should be emphasised that the overall number of analysed cells was large enough to base conclusions on the modification of F-chromatin/interchromatin pattern of lymphocytes of healthy subjects and patients with CLL.

The results of our study indicate that four selected diffractographic descriptors of F-chromatin/interchromatin can precisely differentiate between the 
lymphocytes of healthy subjects and lymphoid cells of CLL patients. The character of F-chromatin/interchromatin modification of lymphoid cells of CLL patients compared with that of lymphocytes of healthy subjects showed that significant dispersion of nuclear content occurred as early as stage $\mathrm{O}$ of development of the disease. As the nucleus is the cellular component that constitutes the principal basis for typing and subtyping of various classes of leukaemias, ${ }^{912}$, we analysed the surface area of nuclear projection of lymphocytes and lymphoid cells and found that the nuclei of the latter were up to $75 \%$ larger. This finding confirms previously reported data. ${ }^{10}$ The enlargement of nuclear size of lymphoid cells in the early stage of CLL development coupled with the dispersion of F-chromatin/interchromatin strongly suggests that there is decondensation of nuclear contents compared with the F-chromatin/interchromatin pattern of lymphocytes of healthy subjects.

The interphase nucleus contains chromatin (stained in this study by the Feulgen method), one or more nucleoli, and interchromatin domains. ${ }^{24}{ }^{25}$ Our results show that in the lymphocytes of healthy subjects and in lymphoid cells of CLL patients the F-chromatin/ interchromatin components of the same size $(380,900$, $2500,3200,4800 \mathrm{~nm}$ ) were evident by light microscopic resolution. On the other hand, the mean relative number of nuclear components of a given size (estimated from the light intensity of diffractographic pattern) was significantly lower for lymphoid cells of CLL patients than for lymphocytes of healthy subjects. The background level of diffractogramsthat is, the density of F-chromatin/interchromatin components of the size below the resolution of light microscope-was twice as large as that of lymphoid cells of CLL patients. Furthermore, in lymphocytes of healthy subjects the F-chromatin/interchromatin components measured on average 380,900 , and 2500 $\mathrm{nm}$ were noted in $80 \%$ of all analysed nuclei. In lymphoid cells of CLL patients, however, components measuring $380 \mathrm{~nm}$ were present in only $40 \%$ of all analysed nuclei; those measuring $900 \mathrm{~nm}$ were present in $60 \%$, and those measuring $2500 \mathrm{~nm}$ in $80 \%$. The finding of a given F-chromatin/interchromatin component size in some (and not in all) nuclei presumably results in optical "dilution" of these components as the background level increased in their vicinity. It is tempting to speculate that the latter resulted in decondensation of F-chromatin/interchromatin followed by formation of components of sizes below the light microscopic resolution. We doubt that the lower light intensity found for the nuclei of lymphoid cells is related to the enlargement of nuclei in chronic leukaemia. The decrease of the mean relative number of nuclear components in a given size of lymphoid cell might be related to enlargement of its nucleus, but we did not observe a decrease in the number of nuclei in which these components occurred. In contrast, the numbers of lymphoid cell nuclei in which the components of a given size could be detected were the same as for normal lymphocytes or even greater.

The results of the present study allow a hypothesis to be made of the structural transition from Fchromatin/interchromatin in lymphocytes of healthy subjects to that of lymphoid cells of CLL patients. The smallest F-chromatin/interchromatin components, measuring on average $380 \mathrm{~nm}$, observed in this study are supposed to be converted to a size below that of the light microscopic resolution. This hypothesis is supported by our findings: the components measuring 380 $\mathrm{nm}$ are less numerous (lower light intensity of diffractographic pattern) in a lymphoid cell nucleus than in a lymphocyte nucleus and they appear in a smaller number of nuclei of the former cells. As the nuclear components measuring $2500 \mathrm{~nm}$ are less numerous in lymphoid cell nuclei than in lymphocyte nuclei, but appear in the same fraction of nuclei of both kinds of cells (the background level around $2500 \mathrm{~nm}$ components failed to increase), we can hypothesise that F-chromatin/interchromatin components measuring $2500 \mathrm{~nm}$ are converted into components of $900 \mathrm{~nm}$ when lymphocytes are converted into lymphoid cells. The latter components are then converted into those sized $380 \mathrm{~nm}$. We found that $900 \mathrm{~nm}$ sized F-chromatin/interchromatin components occurred less often in the nuclei of lymphoid cells than in those of lymphocytes. We can therefore conclude that the transition of nuclear structure from that of a lymphocyte to that of a lymphoid cell of a CLL patient occurs through formation of lower order components of F-chromatin/interchromatin from higher order ones.

Several models of transition from lower to higher order of chromatin organisation have been proposed. ${ }^{26} 27$ Of these, the spiralling, merging, and knotting of chromatin components have been mentioned most often. As we failed to observe the enhancement of background of diffractograms of lymphoid cells, we can at least conclude that the dispersion of chromatin in the latter cells was not the result of simple splitting of components of higher order F-chromatin/interchromatin structures to lower order structures.

The results of this study did not enable us to assess the causes of differences in F-chromatin/interchromatin pattern between lymphocytes of healthy subjects and lymphoid cells of CLL patients. The pattern of fixed and stained chromatin of B lymphocytes was found previously to have characteristics distinct from those of $T$ lymphocytes as indicated by computerised analysis of digitised images of lymphocyte nuclei. ${ }^{28-30}$ In chronic lymphocytic 
leukaemia of B cell type there is a remarkable shift of quantitative proportions between blood $B$ and $T$ lymphocytes compared with those of healthy subjects, ${ }^{15}$ and our results may simply reflect the difference in F-chromatin/interchromatin pattern between $B$ and $T$ lymphocytes. In the population of lymphoid cells of leukaemic patients there are some cycling cells ${ }^{15}$ which are known to be distinct from non-cycling cells as far as their chromatin pattern is concerned. ${ }^{31}$

The results of the present study, although provisional and preliminary due to the small number of blood donors, are encouraging enough to be continued.

This study was supported by CPBR 11.9.69 grant.

\section{References}

1 Eissenberg J, Cartwright IL, Thomas GH, Elgin SCR. Selected topics in chromatin structure. Ann Rev Genet 1985;19:485-536.

2 Sahota TS, Peet FG, Ibaraki A, Farris SH. Chromatin distribution pattern and cell functioning. Can J Zool 1985;64:1908-13.

3 Dardick I, Dardick AM, Jeans MTD, Ripstein P, Caldwell DR. Ultrastructural morphometric image analysis applied to estimation of condensed chromatin in normal and neoplastic lymphocytes. J Electron Microscopy 1985;2:597-609.

4 Komitowski D, Zinser G. Quantitative description of chromatin structure during neoplasia by the method of image processing. Anal Quant Cytol Histol 1985;7:178-82.

5 Young IT, Verbeek PW, Mayall BH. Characterization of chromatin distribution in cell nuclei. Cytometry 1986;7: 467-74.

6 Sawicki W, Rowinski J, Swenson R. Change of chromatin morphology during the cell cycle detected by means of autometed image analysis. J Cell Physiol 1974;84:423-8.

7 Bem W, Rozycka M, Strojny P, Sawicki W. Modification of chromatin pattern in the course of terminal differentiation during human granulocytopoesis: optical diffractometry study. Cell Mol Biol 1987;33:563-71.

8 Setterfield G, Shein R, Dardick I, Kiss G, Dubsky M. Structure of interphase nuclei in relation to the cell cycle. $J$ Cell Biol 1978;77:246-63.

9 Abbot CR, Blewitt RW, Bird CC. Quantitative analysis of nonHodgkin's lymphoma. J Clin Pathol 1982;35:135-8.

10 Crocker J, Jones EL, Curran RC. A comparative study of nuclear form factor, area and diameter in non-Hodgkin's lymphomas and reactive lymph nodes. J Clin Pathol 1983;36:298-302.

11 Lesty C, Raphael M, Nonnenmacher L, et al. An application of mathematical morphology to analysis of the size and shape of nuclei in tissue sections of non-Hodgkin's lymphoma. Cytometry 1986;7:117-31.

12 Van der Valk P, Mosch A, Kurver PJ, Meijer CJLM. Morphometric characterisation of 52 B cell non-Hodgkin's lymphomas. J Clin Pathol 1983;36:289-97.

13 Strojny P, Traczyk Z, Rozycka M, Bem W, Sawicki W. Fourier analysis of nuclear and cytoplasmic shape of blood lymphoid cells from healthy donors and chronic lymphocytic leukemia patients. Anal Quant Cytol Histol 1987;9:475-9.

14 Rai KR, Sawitski A, Cronkite EP, Chanana AD, Levy RN, Pasteumach BS. Clinical studying of chronic lymphocytic leukemia. Blood 1975;46:219-34.

15 Godal T, Funderud S. Human B-cell neoplasms in relation to normal B-cell differentiation and maturation processes. $A d v$ Cancer Res 1982;36:211-55.

16 Boyum A. Separation of leukocytes from blood and bone marrow. Scand J Clin Lab Invest 1968;21(suppl 97):77-81.

17 Culling CFA. Handbook of histopathological and histochemical techniques. 3rd ed. London: Butterworth \& Co, 1974:248-51.

18 Lenczowski S, Rozycka M, Sawicki W, et al. Microscopic image analysis by the optical Fourier transform. Materia Medica Polonia 1980;4:272-85.

19 Pernick B, Kopp RE, Lisa J, Mendelsohn J, Stone H, Wohlers R. Screening of cervical cytological samples using coherent optical processing. Appl Optics 1978;17:21-33.

20 Ostrowski K, Thyberg J, Dziedzic-Goclawska A, et al. Application of optical diffractometry in studies of cell fine structure. Histochem J 1983;78:435-49.

21 Cooley WW, Lohnes PR. Multivariate data analysis. New York: J Wiley \& Sons, 1971.

22 Klecka WR. Discriminant analysis. In: Statistical package for the social sciences. New York: McGraw Hill, 1975:434-67.

23 Casasent D. Coherent optical pattern recognition. Proceedings of the Institute of Electrical and Electronic Engineers 1979;67: 813-25.

24 Krzyzowska-Gruca S, Zborek A, Cruca S. Distribution of interchromatin granules in nuclear matrices obtained from nuclei exhibiting different degrees of chromatin condensation. Cell Tissue Res 1983;231:427-37.

25 Smith HC, Spector DL, Woodcock CLF, Ochs RL, Bhorjee J. Alterations in chromatin conformation are accompanied by reorganization of nonchromatin domains that contain UsnRNP protein p28 and nuclear protein p107. J Cell Biol 1985;101:560-7.

26 Rattner JB, Lin CC. Radial loops and helical coils coexistent in metaphase chromosomes. Cell 1985;42:291-6.

27 Pineta KJ, Coffey DS. A structural analysis of the role of the nuclear matrix and DNA loops in the organization of the nucleus and chromosome. J Cell Sci 1984:(suppl)1:123-35.

28 Durie BGM, Vaught L, Chen YP, Olson GB, Salmon SE, Bartels PH. Discrimination between human T and B Lymphocytes and monocytes by computer analysis of digitized data from scanning microphotometry.I. Chromatin distribution patterns. Blood 1978;51:579-89.

29 Olson GB. Differentiation of murine thoracic duct lymphocytes into $T$ and $B$ subpopulations by computer cell-scanning techniques. Cell Immunol 1974;13:347-55.

30 Renau-Piqueras J, Cerdan F, Barbera E, Cerveta J. Electron microscopic morphometric analysis of human $\mathrm{T}$ and $\mathrm{B}$ peripheral blood lymphocytes. Virchows Arch (Cell Pathol) 1978; 28:47-57.

31 Niccolini C, Giaretti W, De Saive C, Kendall F. The $G_{0}-G_{1}$ transition of WI 38 cells. II. Geometric and densitometric texture analysis. Exp Cell Res 1977;106:119-25.

Requests for reprints to: Dr M Rozycka, Department of Histology, School of Medicine, Chalubinskiego 5, 01-004 Warsaw, Poland. 